PROCEEDINGS OF THE AMERICAN MATHEMATICAL SOCIETY

Volume 124, Number 8, August 1996

\title{
THE INDICES, THE NULLITIES AND THE STABILITY OF TOTALLY GEODESIC SUBMANIFOLDS IN THE COMPLEX QUADRATIC HYPERSURFACES: $Q_{m}=S O(m+2) / S O(m) \times S O(2)$
}

\author{
ZHAO QIANG
}

(Communicated by Roe W. Goodman)

\begin{abstract}
In the paper, the stability of totally geodesic submanfolds in the complex quadratic hypersurfaces: $Q_{m}=S O(m+2) / S O(m) \times S O(2)(m>1)$ is discussed, and the indices, the nullities and the Killing nullities of totally geodesic submanifolds in $Q_{m}$ are calculated.
\end{abstract}

\section{INTRODUCTION}

It is always an interesting and important problem to find all stable minimal submanifolds in each symmetric space. In 1980, B.Y.Chen, P.F.Leung and T.Nagano gave the algorithm of determining the stability of totally geodesic submanifolds in compact symmetric spaces which was reformulated in 1987 by Y.Ohnita (cf. [1] and [2]). In [3], B.Y.Cheng and T.Nagano completely classified complete, connected, totally geodesic submanifolds of the complex quadratic hypersurface: $Q_{m}=S O(m+2) / S O(2) \times S O(m), m>1$. Using their results, we determine the indices and nullities of all totally geodesic submanifolds in $Q_{m}$, and discuss their stability.

Suppose $M$ is a compact minimal submanifold of a Riemannian manifold $N$ with metric $h$. The isometric immersion $\phi: M \rightarrow N$ is called stable if the second derivative of the volume $\operatorname{Vol}\left(M, \phi_{t}^{*} h\right)$ at $t=0$ is nonnegative for every smooth variation $\left\{\phi_{t}\right\}$ of $\phi$ with $\phi_{0}=\phi$. Then we say that $M$ is a stable minimal submanifold of $N$. Choose a smooth variation $\left\{\phi_{t}\right\}$ of $\phi$ with $\phi_{0}=\phi$ and $\left.(\partial / \partial t) \phi_{t}(x)\right|_{t=0}=V_{x}(x \in M)$ for any vector field $V \in \Gamma\left(\phi^{-1} T(N)\right)$. Then the classical second variational formula is given as follows:

$$
\left.\left(d^{2} / d t^{2}\right) \operatorname{Vol}\left(M, \phi_{t}^{*} h\right)\right|_{t=0}=\int_{M}\left\langle\mathcal{J}\left(V^{N}\right), V^{N}\right\rangle d v
$$

where $d v$ denotes the Riemannian measure of $(M, g)$ and $V^{N}$ the component of $V$ normal to $M$. $\mathcal{J}$ is a self-adjoint strongly elliptic linear differential operator of order 2 acting on the space $\Gamma(N(M))$ of smooth sections of the normal bundle $N(M)$, called the Jacobi operator of $\phi$. $\mathcal{J}$ has discrete eigenvalues $\mu_{1}<\mu_{2}<\cdots \rightarrow \infty$. Set

$$
E_{\mu}=\{V \in \Gamma(N(M)) \mid \mathcal{J}(V)=\mu V\} .
$$

Received by the editors August 5, 1994 and, in revised form, January 18, 1995. 1991 Mathematics Subject Classification. Primary 53C35; Secondary 22E70.

Key words and phrases. Indices, nullities, stability. 
We call the number $\sum_{\mu<0} \operatorname{dim} E_{\mu}$ the index of $\phi$ or the index of $M$ in $N$; denote it by $i(\phi)$ or $i(M)$. It is easy to see that $\phi$ is stable if and only if $i(\phi)=0$. The number $\operatorname{dim} E_{0}$ is called the nullity of $\phi$ and denoted by $n(\phi)$ or $n(M)$. Define

$$
P=\left\{X^{N} \mid X \text { is a Killing vector field on } N\right\} \subset \Gamma(N(M)) .
$$

Then $P \subset E_{0}$. The $\operatorname{dim} P$ is called the Killing nullity of $\phi$ and denoted by $n_{k}(\phi)$ or $n_{k}(M)$.

In particular, if $M$ is an $m$-dimensional compact totally geodesic submanifold immersed in a compact Riemannian symmetric space $N$ with metric $g_{N}$, the immersion $\phi: \quad M \rightarrow N$ can be expressed as follows: There are compact symmetric pairs $(U, L)$ and $(G, K)$ with $N=U / L, M=G / K$ and

$$
\begin{aligned}
\phi: M=G / K & \rightarrow N=U / L, \\
g K & \rightarrow \rho(g) L
\end{aligned}
$$

where $\rho: G \rightarrow U$ is an analytic homomorphism with $\rho(K) \subset L$ and the injective differential $\rho: g \rightarrow u$ satisfying $\rho(m) \subset p$. Here $u=l+p$ and $g=k+m$ are the Cartan decompositions of $u$ and $g$, respectively. There exists an $\operatorname{ad} U$-invariant inner product (,) on $u$ such that (,) induces the metric $g_{N}$ on $N$. By (,) we also denote the ad $G$-invariant inner product on $g$ induced from $($,$) through \rho$. Let $m^{\perp}$ be the smooth orthogonal complement of $\rho(m)$ with $p$ relative to (,), and $k^{\perp}$ the orthogonal complement of $\rho(k)$ in $l$. Put $g^{\perp}=k^{\perp}+m^{\perp}$. Then $g^{\perp}$ is the orthogonal complement of $\rho(g)$ in $u$ relative to $($,$) , and g^{\perp}$ is ad $\rho(G)$-invariant. Let $\theta$ be the involutive automorphism of the symmetric pair $(U, L)$. Choose an orthogonal decomposition $g^{\perp}=g_{1}^{\perp} \oplus \cdots \oplus g_{t}^{\perp}$ such that each $g_{i}^{\perp}$ is an irreducible $\operatorname{ad} \rho(G)$-invariant subspace with $\theta\left(g_{i}^{\perp}\right)=g_{i}^{\perp}$. Then the Casimir operator $\mathrm{C}$ of the representation of $G$ on each $g_{i}^{\perp}$ is $a_{i} I$ for $a_{i} \in \mathbf{C}$. Put $g_{i}^{\perp}=k_{i}^{\perp}+m_{i}^{\perp}$, where $k_{i}^{\perp}=k^{\perp} \cap g_{i}^{\perp}$ and $m_{i}^{\perp}=m^{\perp} \cap g_{i}^{\perp}$.

Theorem 1.1 ([1]). The index, nullity and Killing nullity of $\phi$ are given as follows:

$$
\begin{aligned}
& \text { (1) } i(M)=\sum_{i=1}^{t} \sum_{\lambda \in D(G), a_{\lambda}>a_{i}} \operatorname{dim} \operatorname{Hom}_{K}\left(V(\lambda),\left(m_{i}^{\perp}\right)^{\mathbf{C}}\right) \operatorname{dim} V(\lambda) ; \\
& \text { (2) } n(M)=\sum_{i=1}^{t} \sum_{\lambda \in D(G), a_{\lambda}=a_{i}} \operatorname{dim} \operatorname{Hom}_{K}\left(V(\lambda),\left(m_{i}^{\perp}\right)^{\mathbf{C}}\right) \operatorname{dim} V(\lambda) ; \\
& \text { (3) } n_{K}(M)=\sum_{i=1, m_{i}^{\perp} \neq 0}^{t} \operatorname{dim} g_{i}^{\perp},
\end{aligned}
$$

where $D(G)$ is the set of irreducible representations of $G$ and $a_{\lambda}$ is the eigenvalue for the Casimir operator of the irreducible $G$-module $\left(\lambda, V_{\lambda}\right)$ relative to $($,$) .$

\section{The space $Q_{m}$ And its totally Geodesic submanifolds}

The main result of [3] is the following theorem. It gave the classification of all complete, connected, totally geodesic submanifolds of the complex quadratic hypersurface: $Q_{m}=S O(2+m) / S O(2) \times S O(m), m>1$.

Theorem 2.1 ([3]). If $M$ is a maximal totally geodesic submanifold of $Q_{m}, M$ is one of the following three spaces:

(1) $Q_{m-1}$; 
(2) a local Riemannian product of two spheres $S^{p}$ and $S^{q}, p+q=m$;

(3) the complex projective space $P\left(\mathbf{C}^{n+1}\right)$ of complex dimension $n, 2 n=m$.

If $M$ is a nonmaximal, totally geodesic submanifold of $Q_{m}, M$ is either contained in $Q_{m-1}$ in an appropriate position in $Q_{m}$, or the real projective space $P\left(\mathbf{R}^{n+1}\right)$ of real dimension $n, 2 n=m$, which is the intersection of $P\left(\mathbf{C}^{n+1}\right)$ in (3) and the local product space in (2) with $p=q=n$.

Now we have Riemannian symmetric spaces

$$
N=Q_{m}=U / L, \quad U=S O(m+2), \quad L=S O(2) \times S O(m) .
$$

Then $u=l+p$ is the corresponding Cartan decomposition, where

$$
\begin{aligned}
& l=\left\{\left(\begin{array}{cc}
A & 0 \\
0 & B
\end{array}\right) \mid A=\left(\begin{array}{cc}
0 & a \\
-a & 0
\end{array}\right), a \in \mathbf{R}, B \in g l(m), B^{t}=-B\right\} \\
& p=\left\{\left(\begin{array}{cc}
0 & A \\
-A^{t} & 0
\end{array}\right) \mid A \text { is a matrix of } 2 \text { rows and } m \text { columns over } \mathbf{R}\right\} .
\end{aligned}
$$

From now on, we denote the set of matrices of $m$ rows and $n$ columns with elements in $\mathbf{R}$ by $\mathbf{R}(m, n)$.

If $M$ is a compact totally geodesic submanifold immersed in $N$, the immersion $\phi: \quad M \rightarrow N$ can be expressed as follows: There exist a compact symmetric pair $(G, K)$ with $M=G / K$ and a homomorphism $\rho: G \rightarrow U$ such that $\phi$ has form as $g K \rightarrow \rho(g) L$. Then $\rho(K) \subset L$ and the injective differential $\rho: g \rightarrow u$ satisfies $\rho(m) \subset p$. We choose an $\operatorname{ad} U$-invariant inner product on $u$ as $(X, Y)=$ $\operatorname{Tr} X Y(X, Y \in u)$; it induces the metric on $Q_{m}$. Let $g=k+m$ be the corresponding Cartan decomposition of $g$. Below we list the corresponding $\rho, k$ and $m$ in every case.

1. $M=Q_{n}(n<m), \rho: g \rightarrow u$ is

$$
\begin{aligned}
& A \longrightarrow\left(\begin{array}{ll}
A & \\
& 0
\end{array}\right) \in u, \quad A \in g, \\
& k=\left\{\left(\begin{array}{lll}
A & & \\
& B & \\
& & 0
\end{array}\right) \mid A \in g l(2), B \in g l(n), A^{t}=-A, B^{t}=-B\right\}, \\
& m=\left\{\left(\begin{array}{ccc}
0 & A & 0 \\
-A^{t} & 0 & 0 \\
0 & 0 & 0
\end{array}\right) \mid A \in \mathbf{R}(2, n)\right\} .
\end{aligned}
$$

2. $M=S^{p} \times S^{q}(p+q=m) \cdot \rho: g \rightarrow u$ is

$$
(A, B) \longrightarrow\left(\begin{array}{cccccc}
b_{11} & 0 & b_{12} & b_{13} & \cdots & b_{1, q+1} \\
0 & A & 0 & 0 & \cdots & o \\
b_{21} & 0 & b_{22} & b_{23} & \cdots & b_{2, q+1} \\
b_{31} & 0 & b_{32} & b_{33} & \cdots & b_{3, q+1} \\
\cdots & \cdots & \cdots & \cdots & \cdots & \cdots \\
b_{q+1,1} & 0 & b_{q+1,2} & b_{q+1,3} & \cdots & b_{q+1, q+1}
\end{array}\right) .
$$


Here $A=\left(a_{i j}\right) \in g l(p+1), B=\left(b_{i j}\right) \in g l(q+1), A^{t}=-A, B^{t}=-B$.

$$
\begin{aligned}
& k=\left\{\left(\begin{array}{lll}
0 & & \\
& A & \\
& & B
\end{array}\right) \mid A \in g l(p), B \in g l(q), A^{t}=-A, B^{t}=-B\right\}, \\
& m=\left\{\left(\begin{array}{cccc}
0 & 0 & 0 & X \\
0 & 0 & Y & 0 \\
0 & -Y^{t} & 0 & 0 \\
-X^{t} & 0 & 0 & 0
\end{array}\right) \mid X \in \mathbf{R}(1, q), Y \in \mathbf{R}(1, p)\right\} .
\end{aligned}
$$

3. $M=\mathbf{C} P^{n}=S U(n+1) / S U(n)(m=2 n) \cdot \rho: s u(n+1) \rightarrow s o(2 n+2)$ is

$$
A+i B \longrightarrow P_{n+1}(A, B) \text {. }
$$

Here $P_{n+1}(A, B)$ is

$$
\left(\begin{array}{ccccccc}
a_{11} & b_{11} & a_{12} & b_{12} & \cdots & a_{1, n+1} & b_{1, n+1} \\
-b_{11} & a_{11} & -b_{12} & a_{12} & \cdots & -b_{1, n+1} & a_{1, n+1} \\
\cdots & \cdots & \cdots & \cdots & \cdots & \cdots & \cdots \\
a_{n+1,1} & b_{n+1,1} & a_{n+1,2} & b_{n+1,2} & \cdots & a_{n+1, n+1} & b_{n+1, n+1} \\
-b_{n+1,1} & a_{n+1,1} & -b_{n+1,2} & a_{n+1,2} & \cdots & -b_{n+1, n+1} & a_{n+1, n+1}
\end{array}\right)
$$

where $A=\left(a_{i j}\right), B=\left(b_{i j}\right) \in g l(n+1), A^{t}=-A, B^{t}=-B, \operatorname{Tr} B=0$.

$$
\begin{aligned}
& k=\left\{\left(\begin{array}{cc}
0 & 0 \\
0 & P_{n}(B, C)
\end{array}\right) \mid B^{t}=-B, C^{t}=C, \operatorname{Tr} C=0\right\}, \\
& m=\left\{\left(\begin{array}{cc}
0 & O(A, B) \\
-O(A, B)^{t} & 0
\end{array}\right) \mid O(A, B) \in \mathbf{R}(2,2 n)\right\}
\end{aligned}
$$

where

$$
O(A, B)=\left(\begin{array}{ccccc}
a_{1} & b_{1} & \cdots & a_{n} & b_{n} \\
-b_{1} & a_{1} & \cdots & -b_{n} & a_{n}
\end{array}\right) .
$$

4. $M=\mathbf{R} P^{n}=S O(n+1) / S O(n)(m=2 n) . \rho: s o(n+1) \rightarrow s o(2 n+2)$ is $A \rightarrow P_{n+1}(A)$, and $P_{n+1}(A)$ equals

$$
\left(\begin{array}{ccccccc}
a_{11} & 0 & a_{12} & 0 & \cdots & a_{1, n+1} & 0 \\
0 & a_{11} & 0 & a_{12} & \cdots & 0 & a_{1, n+1} \\
\cdots & \cdots & \cdots & \cdots & \cdots & \cdots & \cdots \\
a_{n+1,1} & 0 & a_{n+1,2} & 0 & \cdots & a_{n+1, n+1} & 0 \\
0 & a_{n+1,1} & 0 & a_{n+1,2} & \cdots & 0 & a_{n+1, n+1}
\end{array}\right)
$$

where $A=\left(a_{i j}\right) \in \operatorname{so}(n+1)$.

$$
\begin{aligned}
& k=\left\{\left(\begin{array}{cc}
0 & 0 \\
0 & P_{n}(A)
\end{array}\right) \mid A \in s o(n)\right\} \\
& m=\left\{\left(\begin{array}{cc}
0 & T(A) \\
-T(A)^{t} & 0
\end{array}\right) \mid T(A) \in \mathbf{R}(2,2 n)\right\} \\
& T(A)=\left(\begin{array}{ccccccc}
a_{1} & 0 & a_{2} & 0 & \cdots & a_{n} & 0 \\
0 & a_{1} & 0 & a_{2} & \cdots & 0 & a_{n}
\end{array}\right) .
\end{aligned}
$$




\section{The indices, Nullities And Stability OF THE GEODESIC SUBMANIFOLDS IN $Q_{m}$}

We choose the inner product $(X, Y)=\operatorname{Tr} X Y$ on $u=s o(m+2)$ which induces the metric on $Q_{m}$. In every case, we first calculate $g^{\perp}, m^{\perp}$ and the orthogonal decomposition of the $G$-module $\left(g^{\perp}\right)^{\mathbf{C}}$.

1. $M=Q_{n}(n<m)$,

$$
\begin{aligned}
g^{\perp} & =\left\{\left(\begin{array}{cc}
0 & B \\
-B^{t} & C
\end{array}\right) \mid B \in \mathbf{R}(n+2, m-n), C \in g l(m-n), C^{t}=-C\right\} \\
& =\left\{\left(\begin{array}{cc}
0 & 0 \\
0 & C
\end{array}\right)\right\} \oplus\left\{\left(\begin{array}{cc}
0 & B \\
-B^{t} & 0
\end{array}\right)\right\}=g_{1}^{\perp} \oplus g_{2}^{\perp}, \\
m_{1}^{\perp} & =0 \\
m_{2}^{\perp} & =\left\{\left(\begin{array}{ccc}
0 & 0 & D \\
0 & 0 & 0 \\
-D^{t} & 0 & 0
\end{array}\right) \mid D \in \mathbf{R}(2, m-n)\right\} .
\end{aligned}
$$

Obviously the representation of $G$ on $g_{1}^{\perp}$ is trivial. Below we investigate the $G$ module $\left(g_{2}^{\perp}\right)^{\mathbf{C}}$. Let $E_{i j}$ be the square matrix with entry 1 where the $i$-th row and $j$-th column meet, all other entries being 0 .

1) If $n+2=2 s, m+2=2(s+t)$, set $H_{i}=E_{2 i-1,2 i}-E_{2 i, 2 i-1}(1 \leq i \leq s)$; then $h=\operatorname{span}_{\mathbf{C}}\left\{H_{i}\right\}_{1}^{s}$ is a Cartan subalgebra of $g^{\mathbf{C}}$. Let $F_{i j}=E_{i j}-E_{j i}$ and

$$
G_{j k}^{ \pm}=F_{2 j-1,2 k-1} \pm F_{2 j, 2 k}+i\left(F_{2 j-1,2 k} \mp F_{2 j, 2 k-1}\right)(j \neq k) .
$$

It is obvious that

$$
\begin{aligned}
& \left(g_{2}^{\perp}\right)^{\mathbf{C}}=\operatorname{span}_{\mathbf{C}}\left\{G_{j k}^{ \pm} \quad 1 \leq j \leq s<k \leq s+t \text { or } 1 \leq k \leq s<j \leq s+t\right\}, \\
& (a d H) G_{j k}^{+}=\left(e_{j}(H)-e_{k}(H)\right) G_{j k}^{+}, \\
& (a d H) G_{j k}^{-}=\operatorname{sgn}(j-k)\left(e_{j}(H)+e_{k}(H)\right) G_{j k}^{-}
\end{aligned}
$$

for $H \in h$, where $e_{j}\left(H_{k}\right)=-i \delta_{j k}$. So the set of weights of the $G$-module $\left(g_{2}^{\perp}\right)^{\mathbf{C}}$ is

$$
\Phi=\{\underbrace{ \pm e_{j}, \ldots, \pm e_{j}}_{2 t} \mid 1 \leq j \leq s\} .
$$

We can choose a set of simple roots of $(g)^{\mathbf{C}}$ as

$$
R=\left\{\alpha_{j}=e_{j}-e_{j+1}, \alpha_{s}=e_{s-1}+e_{s} \quad \mid 1 \leq j \leq s\right\} .
$$

In this orientation, all the dominant weights which are also highest weights are $\{\underbrace{e_{1}, \ldots, e_{1}}_{2 t}\}$. But the representation having the highest weight $e_{1}$ is exactly the first basic representation $\omega_{1}$ of $s o(n+2)$, so

$$
\left(g_{2}^{\perp}\right)^{\mathbf{C}}=\underbrace{\omega_{1} \oplus \cdots \oplus \omega_{1}}_{2 t} .
$$

Generally, for the representation having the highest weight $\lambda=\sum_{i} a_{i} \omega_{i}$ of $g$, the Casimir operator $C$ has the form $C=a_{\lambda} I$, where

$$
a_{\lambda}=-\left(a_{i} \omega_{i}+2 \rho, a_{j} \omega_{j}\right)=-\left(a_{i} a_{j} g_{i j}+2 \sum_{j, k} a_{j} g_{j k}\right) .
$$

Here $\rho$ is half the sum of positive roots, and the information about $g_{i j}$ may be found in $[4]$. 
Therefore, the Casimir operator $C$ of $g$ on $\left(g_{i}^{\perp}\right)^{\mathbf{C}}$ has the action $a_{i} I(i=1,2)$; here $a_{1}=0$ and $a_{2}=-(2 s-1)$. So

$$
\begin{aligned}
& \left\{\lambda \in D(G) \quad \mid \quad a_{\lambda}>a_{2}\right\}=\{0\}, \\
& \left\{\lambda \in D(G) \mid a_{\lambda}=a_{2}\right\}=\left\{\omega_{1}\right\} .
\end{aligned}
$$

It is easy to see that $k^{\mathbf{C}}=\mathbf{C} H_{1} \oplus s o(n, \mathbf{C})$. Now $m_{1}^{\perp}=0$, and the set of weights of the $\mathbf{C} H_{1}$-module $\left(m_{2}^{\perp}\right)^{\mathbf{C}}$ is

$$
\{\underbrace{1, \ldots, 1}_{2 t}, \underbrace{-1, \ldots,-1}_{2 t}\} .
$$

Since the action of $s o(n, \mathbf{C})$ on $\left(m_{2}^{\perp}\right)^{\mathbf{C}}$ is trivial, as a $K$-module,

$$
\left(m_{2}^{\perp}\right)^{\mathbf{C}}=\bigoplus_{2 t}(\lambda(1,0, \ldots, 0) \oplus \lambda(-1,0, \ldots, 0)) .
$$

By the branching rule of representations [5], $\operatorname{dim} \omega_{1}=2 s=n+2$,

$$
\omega_{1}= \begin{cases}\lambda(1,1,0, \ldots, 0) \oplus \lambda(1,0, \ldots, 0) \oplus \lambda(-1,0, \ldots, 0), & s>3 \\ \lambda(1,0,1,0, \ldots, 0) \oplus \lambda(1,0, \ldots, 0) \oplus \lambda(-1,0, \ldots, 0), & s=3 .\end{cases}
$$

So we have

$$
\begin{aligned}
i(M) & =0, \\
n(M) & =(n+2)(m-n), \\
n_{K}(M) & =(n+2)(m-n) .
\end{aligned}
$$

2) If $n+2=2 s, m+2=2(s+t)+1$, let

$$
D_{j}^{ \pm}=F_{2 j-1, m+2} \pm i F_{2 j, m+2}, \quad 1 \leq j \leq s .
$$

Then

$$
(a d H) D_{j}^{ \pm}= \pm e_{j}(H) D_{j}^{ \pm}, 1 \leq j \leq s, H \in h .
$$

The set of dominant weights which are also the highest weights of the $g$-module $\left(g_{2}^{\perp}\right)^{\mathbf{C}}$ is

$$
\{\underbrace{\omega_{1}, \ldots, \omega_{1}}_{2 t+1}\}
$$

Using methods similar to 1) we get

$$
\begin{aligned}
i(M) & =0, \\
n(M) & =2 s(2 t+1)=(n+2)(m-n), \\
n_{K}(M) & =(n+2)(m-n) .
\end{aligned}
$$

For other cases of $m+2$ and $n+2$, we can get the same results by similar discussion. 
2. $\phi: M=S^{p} \times S^{q} \longrightarrow Q_{m}(p+q=m)$. By a series of similarity transformations, we have

$$
\begin{aligned}
& g=\left\{\left(\begin{array}{ll}
A & \\
& B
\end{array}\right) \mid A \in \operatorname{so}(p+1), B \in s o(q+1)\right\}, \\
& k=\left\{\left(\begin{array}{cccc}
0 & 0 & 0 & 0 \\
0 & A & 0 & 0 \\
0 & 0 & 0 & 0 \\
0 & 0 & 0 & B
\end{array}\right) \mid A \in s o(p), B \in \operatorname{so}(q)\right\}, \\
& m=\left\{\left(\begin{array}{cccc}
0 & X & 0 & 0 \\
-X^{t} & 0 & 0 & 0 \\
0 & 0 & 0 & Y \\
0 & 0 & -Y^{t} & 0
\end{array}\right) \mid X \in \mathbf{R}(1, p), Y \in \mathbf{R}(1, q)\right\}, \\
& g^{\perp}=\left\{\left(\begin{array}{cc}
0 & B \\
-B^{t} & 0
\end{array}\right) \mid B \in \mathbf{R}(p+1, q+1)\right\}, \\
& m^{\perp}=\left\{\left(\begin{array}{cccc}
0 & 0 & 0 & Y \\
0 & 0 & X & 0 \\
0 & -X^{t} & 0 & 0 \\
-Y^{t} & 0 & 0 & 0
\end{array}\right) \mid X \in \mathbf{R}(1, p), Y \in \mathbf{R}(1, q)\right\} .
\end{aligned}
$$

If $p+1=2 s, q+1=2 t$, choose $H_{i}=E_{2 i-1,2 i}-E_{2 i, 2 i-1}(1 \leq i \leq s+t)$; then $h=\operatorname{span}\left\{H_{i}\right\}_{1}^{s+t}$ is a Cartan subalgebra of $g^{\mathbf{C}}=(s o(2 s) \oplus s o(2 t))^{\mathbf{C}}$. By a similar discussion as before, we know $\left(g^{\perp}\right)^{\mathbf{C}}$ is an irreducible $g$-module. It has the highest weight $\lambda(1,0, \ldots, 0-1,0, \ldots, 0)$, so it is the tensor product of the first basic representations of $s o(2 s)$ and $s o(2 t)$. And

$$
\begin{aligned}
a & =-(\lambda(1,0, \ldots, 0-1,0, \ldots, 0)+2 \rho, \lambda(1,0, \ldots, 0-1,0, \ldots, 0)) \\
& =-\left(\lambda_{1}(1, \underbrace{0, \ldots, 0}_{s-1})+2 \rho_{1}, \lambda_{1}(1,0, \ldots, 0)\right) \\
& -\left(\lambda_{2}(1, \underbrace{0, \ldots, 0}_{t-1})+2 \rho_{2}, \lambda_{2}(1,0, \ldots, 0)\right) \\
& =-2(s+t-1), \\
\{\lambda & \left.\in D(G) \mid a_{\lambda}>a\right\}=\{0, \lambda(1,0, \ldots, 0-0, \ldots, 0), \lambda(0, \ldots, 0-1,0, \ldots, 0)\}, \\
\{\lambda & \left.\in D(G) \mid a_{\lambda}=a\right\}=\{\lambda(1,0, \ldots, 0-1,0, \ldots, 0)\} .
\end{aligned}
$$

Considering $k=s o(p) \oplus s o(q)$, we have

$$
\left(m^{\perp}\right)^{\mathbf{C}}=\left(m_{1}^{\perp}\right)^{\mathbf{C}} \oplus\left(m_{2}^{\perp}\right)^{\mathbf{C}}
$$

as a $K$-module, where

$$
\begin{aligned}
& m_{1}^{\perp}=\left\{\left(\begin{array}{rrr} 
& & 0 \\
-X^{t} & &
\end{array}\right) \mid X \in \mathbf{R}(1, p)\right\}, \\
& m_{2}^{\perp}=\left\{\left(\begin{array}{ccc}
0 & &
\end{array}\right) \mid Y \in \mathbf{R}(1, q)\right\} .
\end{aligned}
$$


It is obvious that the actions of $s o(p)$ on $m_{1}^{\perp}$ and $s o(q)$ on $m_{2}^{\perp}$ are their standard representations. Thus, as a $K$-module,

$$
\left(m^{\perp}\right)^{\mathbf{C}}=\lambda^{\prime}(1,0, \ldots, 0-0, \ldots, 0) \oplus \lambda^{\prime}(0, \ldots, 0-1,0, \ldots, 0) .
$$

But

$$
\begin{aligned}
& \lambda(1,0, \ldots, 0-0, \ldots, 0)=\lambda^{\prime}(0, \ldots, 0-0, \ldots, 0) \oplus \lambda^{\prime}(1,0, \ldots, 0-0, \ldots, 0), \\
& \operatorname{dim} \lambda(1,0, \ldots, 0-0, \ldots, 0)=p+1, \operatorname{dim} \lambda(0, \ldots, 0-1,0, \ldots, 0)=q+1, \\
& \lambda(1,0, \ldots, 0-1,0, \ldots, 0)=\lambda^{\prime}(1, \ldots, 0-1,0, \ldots, 0) \\
& \oplus \lambda^{\prime}(1,0, \ldots, 0-0, \ldots, 0) \oplus \lambda^{\prime}(0, \ldots, 0-1,0, \ldots, 0) \oplus \lambda^{\prime}(0, \ldots, 0-0, \ldots, 0), \\
& \operatorname{dim} \lambda(1,0, \ldots, 0-1,0, \ldots, 0)=(p+1)(q+1) .
\end{aligned}
$$

So we have

$$
\begin{aligned}
& i(M)=m+2, \\
& n(M)=2(p+1)(q+1), \\
& n_{K}(M)=(p+1)(q+1) .
\end{aligned}
$$

We have the same results for the other cases of $p$ and $q$ by similar discussion.

3. $\phi: \mathbf{C} P^{n}=S U(n+1) / S U(n) \longrightarrow Q_{2 n}(m=2 n)$,

$$
\begin{aligned}
g^{\perp} & =\{Q(A, B) \mid A, B \in s o(n+1)\}, \\
m^{\perp} & =\left\{\left(\begin{array}{cc}
0 & s(a, b) \\
-s(a, b)^{t} & 0
\end{array}\right) \mid s(a, b) \in \mathbf{R}(2,2 n)\right\} .
\end{aligned}
$$

Here, for $A=\left(a_{i j}\right), B=\left(b_{i j}\right), Q(A, B)$ is

$$
\begin{gathered}
\left(\begin{array}{ccccccc}
a_{11} & b_{11} & a_{12} & b_{12} & \cdots & a_{1, n+1} & b_{1, n+1} \\
b_{11} & -a_{11} & b_{12} & -a_{12} & \cdots & b_{1, n+1} & -a_{1, n+1} \\
\cdots & \cdots & \cdots & \cdots & \cdots & \cdots & \cdots \\
a_{n+1,1} & b_{n+1,1} & a_{n+1,2} & b_{n+1,2} & \cdots & a_{n+1, n+1} & b_{n+1, n+1} \\
b_{n+1,1} & -a_{n+1,1} & b_{n+1,2} & -a_{n+1,2} & \cdots & b_{n+1, n+1} & -a_{n+1, n+1}
\end{array}\right), \\
s(a, b)=\left(\begin{array}{ccccc}
a_{1} & b_{1} & \cdots & a_{n} & b_{n} \\
b_{1} & -a_{1} & \cdots & b_{n} & -a_{n}
\end{array}\right) .
\end{gathered}
$$

A Cartan subalgebra of $g$ can be imbedded in $u$ as

$$
h=\left\{\sum_{i=1}^{n+1} c_{i} H_{i} \mid \sum_{i} c_{i}=0\right\} .
$$

The set of the weights of the $g$-module $\left(g^{\perp}\right)^{\mathbf{C}}=\sum_{j \neq k} \mathbf{C} G_{j k}^{-}$is

$$
\left\{ \pm\left(e_{j}+e_{k}\right) \mid j \neq k\right\} \text {. }
$$

Choose $\left\{e_{1}-e_{2}, e_{2}-e_{3}, \ldots, e_{n}-e_{n+1}\right\}$ as the set of simple roots of $g^{\mathbf{C}}$. The dominant weights of $\left(g^{\perp}\right)^{\mathbf{C}}$ are $e_{1}+e_{2}$ and $-e_{n}-e_{n+1}$. By comparing the dimensions among $\left(g^{\perp}\right)^{\mathbf{C}}$ and the spaces of the representations having the highest weights $\left\{e_{1}+e_{2},-e_{n}-e_{n+1}\right\}$, we know

$$
\left(g^{\perp}\right)^{\mathbf{C}}=\lambda(0,1,0, \ldots, 0) \oplus \lambda(0, \ldots, 0,1,0) .
$$

Since $\left(m^{\perp}\right)^{\mathbf{C}}=\sum_{k>1}\left(\mathbf{C} G_{1 k}^{-} \oplus \mathbf{C} G_{k 1}^{-}\right)$and $\left\{\sum_{i=2}^{n+1} c_{i} H_{i} \mid \sum_{i} c_{i}=0\right\}$ is a Cartan subalgebra of $k=s u(n)$, the set of the weights of $\left(m^{\perp}\right)^{\mathbf{C}}$ is $\left\{ \pm e_{k}\right\}_{k=2}^{n+1}$. If we choose 
$\left\{e_{i}-e_{i-1} \mid 2 \leq i \leq n\right\}$ as the set of simple roots of $k$, the dominant weights of the $k$-module $\left(m^{\perp}\right)^{\mathbf{C}}$ are $e_{2}$ and $-e_{n+1}$. So

$$
\begin{aligned}
& \left(m^{\perp}\right)^{\mathbf{C}}=\lambda^{\prime}(1,0, \ldots, 0) \oplus \lambda^{\prime}(0, \ldots, 0,1), \\
& \begin{aligned}
a & =-(\lambda(0,1,0, \ldots, 0)+2 \rho, \lambda(0,1,0, \ldots, 0)) \\
& =-(\lambda(0, \ldots, 0,1,0)+2 \rho, \lambda(0, \ldots, 0,1,0)) \\
& =-2(n-1)(n+2) /(n+1),
\end{aligned} \\
& a_{\lambda_{k}}=-k(n-k+1)(n+2) /(n+1) \text { for } \lambda_{k}=\lambda(\underbrace{0, \ldots, 0,1}_{k}, 0, \ldots, 0) ; \\
& \left\{\lambda \in D(G) \mid a_{\lambda}>a\right\}=\left\{0, \lambda_{1}, \lambda_{n}\right\}, \\
& \left\{\lambda \in D(G) \mid a_{\lambda}=a\right\}=\left\{\lambda_{2}, \lambda_{n-1}\right\} .
\end{aligned}
$$

By the branching rule of representations, we know

$$
\begin{aligned}
& \lambda_{1}=\lambda^{\prime}(1,0, \ldots, 0) \oplus \lambda^{\prime}(0, \ldots, 0), \quad \lambda_{n}=\lambda^{\prime}(0, \ldots, 0,1) \oplus \lambda^{\prime}(0, \ldots, 0), \\
& \lambda_{2}=\lambda^{\prime}(0,1,0, \ldots, 0) \oplus \lambda^{\prime}(1,0, \ldots, 0), \\
& \lambda_{n-1}=\lambda^{\prime}(0, \ldots, 0,1) \oplus \lambda^{\prime}(0, \ldots, 0,1,0), \\
& \operatorname{dim} \lambda_{1}=\operatorname{dim} \lambda_{n}=n+1, \quad \operatorname{dim} \lambda_{2}=\operatorname{dim} \lambda_{n-1}=n(n+1) / 2 .
\end{aligned}
$$

Therefore we get

$$
\begin{aligned}
& i(M)=2(n+1), \\
& n(M)=n(n+1), \\
& n_{K}(M)=n(n+1) .
\end{aligned}
$$

4. $\mathbf{R} P^{n}=S O(n+1) / S O(n) \longrightarrow Q_{2 n}(m=2 n)$. Through a series of similarity transformations, we have

$$
\begin{aligned}
& g=\left\{\left(\begin{array}{ll}
A & \\
& A
\end{array}\right) \mid A \in \operatorname{so}(n+1)\right\}, \\
& k=\left\{\left(\begin{array}{cccc}
0 & & & \\
& A & & \\
& & 0 & \\
& & & A
\end{array}\right) \mid A \in \operatorname{so}(n)\right\}, \\
& m=\left\{\left(\begin{array}{cccc}
0 & X & 0 & 0 \\
-X^{t} & 0 & 0 & 0 \\
0 & 0 & 0 & X \\
0 & 0 & -X^{t} & 0
\end{array}\right) \mid X \in \mathbf{R}(1, n)\right\} \text {, } \\
& g^{\perp}=g_{1}^{\perp} \oplus g_{2}^{\perp} \oplus g_{3}^{\perp} \text {, } \\
& m^{\perp}=m_{1}^{\perp} \oplus m_{2}^{\perp} \oplus m_{3}^{\perp} \text {, }
\end{aligned}
$$


where

$$
\begin{aligned}
& g_{1}^{\perp}=\left\{\left(\begin{array}{cc}
C & \\
& -C
\end{array}\right) \mid C \in \operatorname{so}(n+1)\right\}, \\
& g_{2}^{\perp}=\left\{\left(\begin{array}{cc}
0 & B \\
-B^{t} & 0
\end{array}\right) \mid B=B^{t} \in g l(n+1)\right\} \text {, } \\
& g_{3}^{\perp}=\left\{\left(\begin{array}{cc}
0 & B \\
-B^{t} & 0
\end{array}\right) \mid B \in s o(n+1)\right\} \text {, } \\
& m_{1}^{\perp}=0, m_{2}^{\perp}=\left\{\left(\begin{array}{cccc} 
& & & \\
& & X^{t} & \\
-X^{t} & & &
\end{array}\right) \mid X \in \mathbf{R}(1, n)\right\} \text {, } \\
& m_{3}^{\perp}=\left\{\left(\begin{array}{lll} 
& & X^{t} \\
& -X &
\end{array}\right) \mid X \in \mathbf{R}(1, n)\right\} .
\end{aligned}
$$

It is easy to see

$$
\left(g_{2}^{\perp}\right)^{\mathbf{C}}=\left(g_{21}^{\perp}\right)^{\mathbf{C}} \oplus\left(g_{22}^{\perp}\right)^{\mathbf{C}},
$$

where

$$
\begin{aligned}
& \left(g_{21}^{\perp}\right)^{\mathbf{C}}=\lambda(0, \ldots, 0), \quad\left(g_{22}^{\perp}\right)^{\mathbf{C}}=\lambda(2,0, \ldots, 0), \\
& \left(m_{21}^{\perp}\right)^{\mathbf{C}}=0, \quad\left(m_{22}^{\perp}\right)^{\mathbf{C}}=\left(m_{2}^{\perp}\right)^{\mathbf{C}} .
\end{aligned}
$$

Without loss of generality, suppose $n=2 s-1$; then

$$
\left(g_{1}^{\perp}\right)^{\mathbf{C}}=\lambda(0,1,0, \ldots, 0),\left(g_{3}^{\perp}\right)^{\mathbf{C}}=\lambda(0,1,0, \ldots, 0) .
$$

As a $K$-module, it is easy to see

$$
\left(m_{2}^{\perp}\right)^{\mathbf{C}}=\lambda^{\prime}(1,0, \ldots, 0), \quad\left(m_{3}^{\perp}\right)^{\mathbf{C}}=\lambda^{\prime}(1,0, \ldots, 0) .
$$

So $a_{21}=-(4 s-2), a_{3}=-4(s-1)$. For $\lambda_{k}=\lambda(\underbrace{0, \ldots, 0,1}_{k}, 0, \ldots, 0)$, we have

$$
\begin{aligned}
& a_{\lambda_{k}}=-k(2 s-k)(1 \leq k \leq s-2), a_{\lambda_{s}}=a_{\lambda_{s-1}}=-s^{2} / 2 . \\
& \left\{\lambda \in D(G) \mid a_{\lambda}>a_{21}\right\}= \begin{cases}\left\{\lambda_{1}, \lambda_{2}\right\}, & s>8, \\
\left\{\lambda_{1}, \lambda_{2}, \lambda_{s-1}, \lambda_{s}\right\}, & 3 \leq s \leq 7, \\
\left\{\lambda_{1}, \lambda_{2}, \lambda_{3}\right\}, & s=3,\end{cases} \\
& \left\{\lambda \in D(G) \mid a_{\lambda}=a_{21}\right\}=\lambda(2,0, \ldots, 0), \\
& \left\{\lambda \in D(G) \mid a_{\lambda}>a_{3}\right\}= \begin{cases}\lambda_{1}, & s>6, \\
\left\{\lambda_{1}, \lambda_{s-1}, \lambda_{s}\right\}, & s \leq 6,\end{cases} \\
& \left\{\lambda \in D(G) \mid a_{\lambda}=a_{3}\right\}=\lambda_{2} .
\end{aligned}
$$


By the branching rule of representations, if $s>3$ we get

$$
\begin{aligned}
& \lambda_{1}=\lambda^{\prime}(1,0, \ldots, 0) \oplus \lambda^{\prime}(0, \ldots, 0), \operatorname{dim} \lambda_{1}=n+1, \\
& \lambda(2,0, \ldots, 0)=\lambda^{\prime}(2,0, \ldots, 0) \oplus \lambda^{\prime}(1,0, \ldots, 0) \oplus \lambda^{\prime}(0, \ldots, 0), \\
& \operatorname{dim} \lambda(2,0, \ldots, 0)=\frac{(n+1)(n+2)}{2}-1, \\
& \lambda_{2}=\lambda^{\prime}(0,1,0, \ldots, 0) \oplus \lambda^{\prime}(1,0, \ldots, 0), \operatorname{dim} \lambda_{2}=n(n+1) / 2, \\
& \lambda_{s}=\lambda^{\prime}(0, \ldots, 0,1)=\lambda_{s-1}, \\
& \operatorname{dim} \lambda_{s-1}=\operatorname{dim} \lambda_{s}=2^{s-1}
\end{aligned}
$$

If $s=3$

$$
\begin{aligned}
& \lambda(2,0,0)=\lambda^{\prime}(2,0), \quad \lambda_{1}=\lambda^{\prime}(1,0)=\lambda_{3}, \\
& \lambda_{2}=\lambda^{\prime}(0,0) \oplus \lambda^{\prime}(0,1), \\
& \operatorname{dim} \lambda(2,0,0)=10, \operatorname{dim} \lambda_{1}=\operatorname{dim} \lambda_{3}=4, \operatorname{dim} \lambda_{2}=6 .
\end{aligned}
$$

Thus we have

$$
\begin{aligned}
& i(M)= \begin{cases}(n+1)(n+4) / 2, & n>5, \\
16, & n=5,\end{cases} \\
& n(M)= \begin{cases}(n+1)^{2}-1, & n>5, \\
0, & n=5,\end{cases} \\
& n_{K}(M)=(n+1)^{2}-1 .
\end{aligned}
$$

Finally, we get the following theorem:

Theorem 3.1. The indices, the nullities and the Killing nullities of the totally geodesic submanifolds in $Q_{m}$ are listed in the following table. Among all the totally geodesic submanifolds in $Q_{m}$, only $Q_{n}(n<m)$ are stable; the nullity and the Killing nullity are equivalent except for $S^{p} \times S^{q}(p+q=m)$.

\begin{tabular}{||c|c|c|c||}
\hline$M$ & $i(M)$ & $n(M)$ & $n_{K}(M)$ \\
\hline$Q_{n}$ & 0 & $(n+2)(m-n)$ & $(n+2)(m-n)$ \\
\hline$S^{P} \times S^{q}(p+q=m)$ & $m+2$ & $2(p+1)(q+1)$ & $(p+1)(q+1)$ \\
\hline $\mathbf{C} P^{n}(m=2 n)$ & $2(n+1)$ & $n(n+1)$ & $n(n+1)$ \\
\hline $\mathbf{R} P^{n}(m=2 n>10)$ & $(n+1)(n+4) / 2$ & $(n+1)^{2}-1$ & $(n+1)^{2}-1$ \\
\hline
\end{tabular}

\section{REFERENCES}

1. Y.Ohnita, On stability of minimal submanifolds in compact symmetric spaces, Compsitio Math., 64(1987), 157-189. MR 88k:53082

2. B.Y.Chen, Geometry of slant submanifolds, Katholieke Universiteit Leuven, 1990. MR 92d:53047

3. B.Y.Chen and T.Nagano, Totally geodesic submanifolds of symmetric spaces I, Duke Math. J., 44(1977), 745-755. MR 56:16543

4. E.B.Dynkin, Semisimple subalgebras of semisimple Lie algebras, Amer. Math. Soc. Transl. Ser. 2, vol. 6, Amer. Math. Soc., Providence, RI, 1957, pp. 111-244. (Russian original) MR 13:904c 
5. W.G.Mckay and J.Patera, Tables of dimensions, indices, and branching rules for representations of simple Lie algebras, Lecture Notes in Pure and Applied Mathematics, vol. 69, Marcel Dekker, New York and Basel, 1981. MR 82i:17008

Department of Mathematics, Beijing University, Beijing, 100871, People's Republic OF CHINA

Department of Mathematics, Northwest Normal University, Lanzhou, 730070, PeoPLE'S REPUBLIC OF CHINA 